\title{
SOME ISOMORPHISMS IN DERIVED FUNCTORS AND THEIR APPLICATIONS
}

\author{
K. KHASHYARMANESH ${ }^{\bowtie}$ and F. KHOSH-AHANG
}

(Received 18 December 2010; accepted 17 October 2012; first published online 15 May 2013)

\author{
Communicated by B. Gardner
}

\begin{abstract}
Let $R$ be a commutative Noetherian ring, $M$ be a finitely generated $R$-module and a be an ideal of $R$ such that $\mathrm{a} M \neq M$. We show among the other things that, if $c$ is a nonnegative integer such that $H_{\mathfrak{a}}^{i}(M)=0$ for all $i<c$, then there is an isomorphism $\operatorname{End}\left(H_{\mathfrak{a}}^{c}(M)\right) \cong \operatorname{Ext}_{R}^{c}\left(H_{\mathfrak{a}}^{c}(M), M\right)$; and if $c$ is a nonnegative integer such that $H_{\mathfrak{a}}^{i}(M)=0$ for all $i \neq c$, there are the following isomorphisms:

(i) $H_{\mathfrak{b}}^{i}\left(H_{\mathfrak{a}}^{c}(M)\right) \cong H_{\mathfrak{b}}^{i+c}(M)$ and

(ii) $\operatorname{Ext}_{R}^{i}\left(R / \mathfrak{b}, H_{\mathfrak{a}}^{c}(M)\right) \cong \operatorname{Ext}_{R}^{i+c}(R / \mathfrak{b}, M)$

for all $i \in \mathbb{N}_{0}$ and all ideals $\mathfrak{b}$ of $R$ with $\mathfrak{b} \supseteq \mathfrak{a}$. We also prove that if $\mathfrak{a}$ and $\mathfrak{b}$ are ideals of $R$ with $\mathfrak{b} \supseteq \mathfrak{a}$ and $c:=\operatorname{grade}(\mathfrak{a}, M)$, then there exists a natural homomorphism from $\operatorname{End}\left(H_{\mathfrak{a}}^{c}(M)\right)$ to $\operatorname{End}\left(H_{\mathfrak{b}}^{c}(M)\right)$, where grade $(\mathfrak{a}, M)$ is the maximum length of $M$-sequences in $\mathfrak{a}$.
\end{abstract}

2010 Mathematics subject classification: primary 13D45; secondary 13D07.

Keywords and phrases: local cohomology module, cohomological dimension, Čech complex, cohomological complete intersection.

\section{Introduction}

Let $R$ be a commutative Noetherian ring and $M$ be an $R$-module. Let $C(R)$ be the category of all modules and homomorphisms over $R$. Also, for a nonnegative integer $n$, we denote the $n$th local cohomology functor with support in an ideal a of $R$ by $H_{\mathfrak{a}}^{n}(-)$. In addition, we denote the cohomological dimension of $M$ with respect to $\mathfrak{a}$ by

$$
\operatorname{cd}(\mathfrak{a}, M)\left(=\sup \left\{i \in \mathbb{Z} \mid H_{\mathfrak{a}}^{i}(M) \neq 0\right\}\right) .
$$

Let $\mathfrak{a}$ be an ideal of a commutative Noetherian $\operatorname{ring} R$ and $M$ be a finitely generated $R$-module such that $\mathfrak{a} M \neq M$ and $\operatorname{grade}(\mathfrak{a}, M)$ denote the maximum length of $M$ sequences in $\mathfrak{a}$. There are some attempts to describe the module $H_{\mathfrak{a}}^{c}(M)$, where $c:=$

The first author was supported by a grant from Ferdowsi University of Mashhad (No. MP89179KHA).

(c) 2013 Australian Mathematical Publishing Association Inc. 1446-7887/2013 \$16.00 
$\operatorname{grade}(\mathfrak{a}, M)$. For instance, it is well known that $\operatorname{Ass}_{R}\left(H_{\mathfrak{a}}^{c}(M)\right)=\operatorname{Ass}\left(\operatorname{Ext}_{R}^{c}(R / \mathfrak{a}, M)\right)$. On the other hand, an ideal $\mathfrak{a}$ of $R$ is called a cohomological complete intersection if height ${ }_{R} \mathfrak{a}=\operatorname{cd}(\mathfrak{a}, R)$. Recently there have been some interesting results about the cohomological complete intersection ideals over a Gorenstein ring (cf. [3, 4, 8]). Note that when $R$ is Gorenstein, $\mathfrak{a}$ is cohomological complete intersection if $\operatorname{grade}(\mathfrak{a}, R)=$ $\operatorname{cd}(\mathfrak{a}, R)$.

Suppose that $F$ is an $R$-linear functor from $C(R)$ to itself such that for every $R$ module $L, F(L)$ is a-torsion. For each $i \in \mathbb{N}_{0}$, we use $\mathcal{R}^{i} F(-)$ to denote the $i$ th right derived functor of $F$. It is natural to ask about the module $\mathcal{R}^{c} F(M)$ when $c=\operatorname{grade}(\mathfrak{a}, M)$ or $c=\operatorname{grade}(\mathfrak{a}, M)=\operatorname{cd}(\mathfrak{a}, M)$. In the second section of this paper we prove, for a nonnegative integer $c$, that:

(i) $\quad$ if $c \leqslant \operatorname{grade}(\mathfrak{a}, M)$, then $\mathcal{R}^{0} F\left(H_{\mathfrak{a}}^{c}(M)\right) \cong \mathcal{R}^{c} F(M)$; and

(ii) $\quad$ if $c:=\operatorname{cd}(\mathfrak{a}, M)=\operatorname{grade}(\mathfrak{a}, M)$, then $\mathcal{R}^{i} F\left(H_{\mathfrak{a}}^{c}(M)\right) \cong \mathcal{R}^{i+c} F(M)$ for all $i \in \mathbb{N}_{0}$.

Whenever $(R, \mathrm{~m})$ is a Gorenstein local ring, Hellus and Schenzel showed in [4] that if $\mathfrak{a}$ is a complete intersection ideal of $R$ in $V(\mathfrak{a}) \backslash\{\mathfrak{m}\}, c:=\operatorname{cd}(\mathfrak{a}, R)$ and $d:=\operatorname{dim}_{R} R / \mathfrak{a}$, then the following conditions are equivalent:

(i) $\quad \mathfrak{a}$ is a cohomological complete intersection;

(ii) $H_{\mathfrak{m}}^{d}\left(H_{\mathfrak{a}}^{c}(R)\right) \cong E(R / \mathfrak{m})$ and $H_{\mathfrak{m}}^{i}\left(H_{\mathfrak{a}}^{c}(R)\right)=0$ for all $i \neq d$, where $E(R / \mathfrak{m})$ is the injective hull of the residue field $R / \mathrm{m}$;

(iii) $\operatorname{dim}_{R / \mathfrak{m}} \operatorname{Ext}_{R}^{i}\left(R / \mathrm{m}, H_{\mathfrak{a}}^{c}(R)\right)=\delta_{d, i}$.

(See [4, Theorems 0.1 and 3.2] for other equivalent conditions.) To do this, by using a minimal injective resolution of a Gorenstein local ring $R$, they first introduced a new complex which is called the truncation complex, and then applied spectral sequence methods. In the third section of the present paper, by using the Čech complex, we provide the following theorem.

THEOREM 1.1. Let $\mathfrak{a}$ be an arbitrary ideal of a commutative Noetherian ring $R$ and $M a$ finitely generated $R$-module such that $\mathfrak{a} M \neq M$ and $c:=\operatorname{cd}(\mathfrak{a}, M)=\operatorname{grade}(\mathfrak{a}, M)$. Then, for every ideal $\mathfrak{b}$ of $R$ with $\mathfrak{b} \supseteq \mathfrak{a}$ :

(i) $H_{\mathfrak{b}}^{i}\left(H_{\mathfrak{a}}^{c}(M)\right) \cong H_{\mathfrak{b}}^{i+c}(M)$ and

(ii) $\operatorname{Ext}_{R}^{i}\left(R / \mathfrak{b}, H_{\mathfrak{a}}^{c}(M)\right) \cong \operatorname{Ext}_{R}^{i+c}(R / \mathfrak{b}, M)$

for all $i \in \mathbb{N}_{0}$.

We also show that [4, Corollary 2.3 and Lemma 2.6] are consequences of Theorem 1.1.

Whenever $R$ is a complete local ring, Hellus and Stückrad showed in [5] that if a is a cohomological complete intersection with $c:=\operatorname{cd}(\mathfrak{a}, R)$, then the endomorphism ring $\operatorname{End}\left(H_{\mathfrak{a}}^{c}(R)\right)$ is isomorphic to $R$ (see also [6, Theorem 2.7]). Schenzel [8] gave a characterization when the endomorphism ring $\operatorname{End}\left(H_{\mathfrak{a}}^{c}(R)\right)$ is isomorphic to $R$. Also, he proved that if $\mathfrak{a}$ and $\mathfrak{b}$ are ideals of a Gorenstein local ring $R$ such that $\mathfrak{b} \supseteq \mathfrak{a}$ and $c:=$ height $_{R} \mathfrak{a}=$ height $_{R} \mathfrak{b}$, then there exists a natural homomorphism $\operatorname{End}\left(H_{\mathfrak{a}}^{c}(R)\right) \longrightarrow \operatorname{End}\left(H_{\mathfrak{b}}^{c}(R)\right)$. In the final section, we not only provide an improved 
form of [8, Lemma 2.3(d)] and [6, Theorem 2.7] but also prove the following theorem which is a generalization of [8, Theorem 1.2(a)].

THEOREM 1.2. Let $\mathfrak{a}$ and $\mathfrak{b}$ be ideals of an arbitrary commutative Noetherian ring $R$ and $M$ a finitely generated $R$-module such that $\mathfrak{b} \supseteq \mathfrak{a}, \mathfrak{a} M \neq M$ and $c:=\operatorname{grade}(\mathfrak{a}, M)$. Then:

(i) we have a monomorphism from $H_{\mathfrak{b}}^{c}(M)$ to $H_{\mathfrak{a}}^{c}(M)$; and

(ii) there exists a natural homomorphism from $\operatorname{End}\left(H_{\mathfrak{a}}^{c}(M)\right)$ to $\operatorname{End}\left(H_{\mathfrak{b}}^{c}(M)\right)$.

We would like to give two different proofs of our results. The first of them is, in fact, a modification of that we have exploited in [6]. The second proof, which is of a completely different nature, is based on a special spectral sequence.

Throughout this paper, $R$ will denote a commutative Noetherian ring with nonzero identity, a an ideal of $R$ and $M$ a finitely generated $R$-module. We shall use $\mathbb{N}_{0}$ (respectively, $\mathbb{N}$ ) to denote the set of nonnegative (respectively, positive) integers. Also, when $F$ is a covariant $R$-linear functor from $C(R)$ to itself, we adopt the convention that, for every negative integer $i, \mathcal{R}^{i} F(-)$ is the zero functor. Our terminology follows Brodmann and Sharp's textbook [2] on local cohomology.

\section{Derived functors and Čech complex}

We begin with the following lemma. Note that, for an $R$-module $N$ and element $x$ in $R$, we use the notation $N_{x}$ for the module of fractions of $N$ with respect to the multiplicatively closed subset $\left\{x^{u} \mid u \in \mathbb{N}_{0}\right\}$ of $R$.

LEMMA 2.1. Let $F$ be a covariant (or contravariant) functor from $C(R)$ to itself such that, for every $R$-module $L, F(L)$ is a-torsion, and let $x$ be an arbitrary element of $\mathfrak{a}$. Then, for every $R$-module $N, \mathcal{R}^{i} F\left(N_{x}\right)=0$ for all $i \in \mathbb{N}_{0}$.

Proof. Suppose that $x \in \mathfrak{a}$ and $N$ is an $R$-module. Let $g: N_{x} \longrightarrow N_{x}$ be the map given by multiplication by $x$. It is easy to see that $g$ is an automorphism on $N_{x}$. Hence, for all $i \in \mathbb{N}_{0}, \mathcal{R}^{i} F(g): \mathcal{R}^{i} F\left(N_{x}\right) \stackrel{x}{\longrightarrow} \mathcal{R}^{i} F\left(N_{x}\right)$ is again an automorphism provided by multiplication by $x$. Now, since, by our assumption, for an $R$-module $L$, every element of $F(L)$ is annihilated by some power of $x$, it is routine to check that $\mathcal{R}^{i} F\left(N_{x}\right)$ is zero.

Note that, similar to the proof of Lemma 2.1, one can show that if $T$ is an a-torsion $R$-module, then for every $R$-module $N, \operatorname{Ext}_{R}^{i}\left(T, N_{x}\right)=0$ for all $i \in \mathbb{N}_{0}$. In this regard, the result of Lemma 2.1 holds true for the functor $\operatorname{Hom}_{R}(T,-)$, where $T$ is an a-torsion $R$-module.

Notation 2.2. Let $\underline{\mathrm{x}}:=x_{1}, \ldots, x_{n}$ be a sequence of elements of $R$ and $N$ be an $R$ module. Set $\mathfrak{b}:=\left(x_{1}, \ldots, x_{n}\right)$. Recall that the Čech complex $C(\underline{x}, N)^{\bullet}$ of $N$ with respect to $\underline{x}$ is the complex

$$
0 \longrightarrow C^{0} \longrightarrow C^{1} \longrightarrow \cdots \longrightarrow C^{i} \stackrel{d^{i}}{\longrightarrow} C^{i+1} \longrightarrow \cdots \longrightarrow C^{n} \longrightarrow 0,
$$

where $C^{0}=N$ and, for $1 \leqslant i \leqslant n, C^{i}$ is a direct sum of some copies of $N_{x_{k(1)} \ldots x_{k(i)}}$ where $1 \leqslant k(1)<k(2)<\cdots<k(i) \leqslant n$ and $C^{i}$ is zero elsewhere (cf. [2, Proposition 
and Definition 5.1.5]). Also note that, by [2, Theorem 5.1.19], $H^{i}\left(C(\underline{\mathrm{x}}, N)^{\bullet}\right) \cong H_{\mathrm{b}}^{i}(N)$ for all $i \in \mathbb{N}_{0}$. Throughout this section, we set $L^{i}:=\operatorname{Im} d^{i}$ and $K^{i}:=\operatorname{Ker} d^{i}$ for all $i \in \mathbb{Z}$.

Theorem 2.3 (Compare [6, Theorem 2.6], [7, Theorem 1.1(v)] and [8, Lemma 2.2(d)]). Let $F$ be an R-linear covariant functor from $C(R)$ to itself such that for every $R$-module $L, F(L)$ is a-torsion. Also let $c \in \mathbb{N}_{0}$ and $\mathfrak{a}$ be an ideal of $R$ such that $\mathfrak{a} M \neq M$ and that $c \leqslant \operatorname{grade}(\mathfrak{a}, M)$. Then

$$
\mathcal{R}^{0} F\left(H_{\mathfrak{a}}^{c}(M)\right) \cong \mathcal{R}^{c} F(M) .
$$

FIRST PROOF. Let $\underline{x}:=x_{1}, \ldots, x_{n}$ be a sequence of elements of $R$ which generates the ideal a. Then, in view of [2, Corollary 3.3.3 and Theorem 6.2.7], we have that $n \geqslant c$. Now, consider the Čech complex of $M$ with respect to $\underline{\mathrm{x}}$ as follows:

$$
C(\underline{\mathrm{x}}, M)^{\bullet}: 0 \longrightarrow C^{0} \stackrel{d^{0}}{\longrightarrow} C^{1} \longrightarrow \cdots \longrightarrow C^{c} \stackrel{d^{c}}{\longrightarrow} C^{c+1} \longrightarrow \cdots \longrightarrow C^{n} \stackrel{d^{n}}{\longrightarrow} 0 .
$$

So, we have the exact sequence

$$
0 \longrightarrow K^{i} \longrightarrow C^{i} \longrightarrow L^{i} \longrightarrow 0
$$

which, in light of Lemma 2.1, induces the isomorphism

$$
\mathcal{R}^{j} F\left(K^{i}\right) \cong \mathcal{R}^{j-1} F\left(L^{i}\right),
$$

for all $j \in \mathbb{N}_{0}$ and $i \neq 0$. Now, by [2, Theorem 5.1.19], we have the exact sequence

$$
0 \longrightarrow L^{i-1} \longrightarrow K^{i} \longrightarrow H_{\mathfrak{a}}^{i}(M) \longrightarrow 0
$$

for all $i \in \mathbb{N}_{0}$. So, applying the long exact sequence $\mathcal{R}^{j} F(-)$ on (2.3), together with (2.2), yields the exact sequence

$$
\mathcal{R}^{j-2} F\left(L^{i}\right) \longrightarrow \mathcal{R}^{j-1} F\left(H_{\mathfrak{a}}^{i}(M)\right) \longrightarrow \mathcal{R}^{j} F\left(L^{i-1}\right) \longrightarrow \mathcal{R}^{j-1} F\left(L^{i}\right)
$$

for all $j \in \mathbb{N}_{0}$ and $i \neq 0$. Using (2.4) for $j:=0$ implies that

$$
\mathcal{R}^{0} F\left(L^{i}\right)=0 \quad \text { for all } i \in \mathbb{N}_{0} .
$$

So, using again of the exact sequence (2.4) for $j:=1$ and $i:=c$ ensures the isomorphism

$$
\mathcal{R}^{0} F\left(H_{\mathfrak{a}}^{c}(M)\right) \cong \mathcal{R}^{1} F\left(L^{c-1}\right) .
$$

On the other hand, since $c \leqslant \operatorname{grade}(\mathfrak{a}, M)$, when $c \neq 0$, we have the exact sequences

$$
0 \longrightarrow M \longrightarrow C^{1} \longrightarrow L^{1} \longrightarrow 0
$$

and

$$
0 \longrightarrow L^{j-1} \longrightarrow C^{j} \longrightarrow L^{j} \longrightarrow 0
$$


for all $2 \leqslant j \leqslant c-1$. Hence, in view of Lemma 2.1, we have the isomorphisms

$$
\begin{aligned}
\mathcal{R}^{c} F(M) & \cong \mathcal{R}^{c-1} F\left(L^{1}\right) \\
& \cong \mathcal{R}^{c-2} F\left(L^{2}\right) \\
& \cong \cdots \\
& \cong \mathcal{R}^{2} F\left(L^{c-2}\right) \\
& \cong \mathcal{R}^{1} F\left(L^{c-1}\right) .
\end{aligned}
$$

Now, the isomorphism (2.6) completes the proof, for all $c \neq 0,1$. When $c=0$, the exact sequence (2.3) yields the isomorphism

$$
\mathcal{R}^{0} F\left(H_{\mathfrak{a}}^{0}(M)\right) \cong \mathcal{R}^{0} F\left(K^{0}\right) .
$$

Therefore (2.1), (2.5) and (2.7) ensure that $\mathcal{R}^{0} F\left(H_{\mathrm{a}}^{0}(M)\right) \cong \mathcal{R}^{0} F(M)$ as desired. Also, when $c=1$, since $H_{\mathfrak{a}}^{0}(M)=0$, [2, Theorem 5.1.19] implies that $L^{0}=C^{0}=M$. So, the result follows from the isomorphism (2.6).

SECOND PRoOF. Since $F(L)$ is a-torsion for every $R$-module $L$, there is an isomorphism of derived functors

$$
\mathcal{R} F \cong \mathcal{R}\left(\Gamma_{\mathfrak{a}} \cdot F\right) \cong \mathcal{R}\left(F \cdot \Gamma_{\mathfrak{a}}\right) \cong \mathcal{R} F \cdot \mathcal{R} \Gamma_{\mathfrak{a}} .
$$

Hence we have a spectral sequence

$$
E_{2}^{p, q}=\mathcal{R}^{p} F\left(H_{\mathfrak{a}}^{q}(M)\right) \Longrightarrow \mathcal{R}^{p+q} F(M) .
$$

Since $c \leqslant \operatorname{grade}(\mathfrak{a}, M)$, we have $H_{\mathfrak{a}}^{q}(M)=0$ if $q<c$. Thus we obtain an isomorphism $\mathcal{R}^{0} F\left(H_{\mathfrak{a}}^{c}(M)\right)=E_{2}^{0, c} \cong \mathcal{R}^{c} F(M)$.

The following corollary is an immediate consequence of Theorem 2.3 and [2, Theorem 6.2.7].

Corollary 2.4. Let $\mathfrak{a}$ be an ideal of $R$ such that $\mathfrak{a} M \neq M$ and $c:=\operatorname{grade}(\mathfrak{a}, M)$. Suppose that $F$ is an R-linear covariant functor such that for each R-module $L, F(L)$ is a-torsion. Then:

(i) $\mathcal{R}^{j} F(M)$ is zero for all $j<c$ and $\mathcal{R}^{c} F(M) \cong \mathcal{R}^{0} F\left(H_{\mathfrak{a}}^{c}(M)\right)$; and

(ii) if $\mathfrak{b}$ is another ideal of $R$ such that $\mathfrak{b} M \neq M$ and $F(L)$ is also b-torsion for all $R$-modules $L$, then for every nonnegative integer $k$, provided that $k \leqslant$ $\inf \{\operatorname{grade}(\mathfrak{a}, M)$, grade $(\mathfrak{b}, M)\}$, we have the isomorphism

$$
\mathcal{R}^{0} F\left(H_{\mathfrak{a}}^{k}(M)\right) \cong \mathcal{R}^{0} F\left(H_{\mathfrak{b}}^{k}(M)\right) .
$$

The following theorem is one of the main results of this paper. 
THEOREM 2.5. Let $F$ be an $R$-linear covariant functor from $C(R)$ to itself such that for every $R$-module $L, F(L)$ is $\mathfrak{a}$-torsion. Suppose that $\mathfrak{a}$ is an ideal of $R$ and $M$ is a finitely generated $R$-module such that $\mathfrak{a} M \neq M$ and that $c:=\operatorname{cd}(\mathfrak{a}, M)=\operatorname{grade}(\mathfrak{a}, M)$. Then

$$
\mathcal{R}^{i} F\left(H_{\mathfrak{a}}^{c}(M)\right) \cong \mathcal{R}^{i+c} F(M)
$$

for all $i \in \mathbb{N}_{0}$.

FiRST PROOF. Let $\underline{x}:=x_{1}, \ldots, x_{n}$ be a sequence of elements of $R$ which generates the ideal a. Then, in view of [2, Corollary 3.3.3], we have that $n \geqslant c$. Now, consider the Čech complex of $M$ with respect to $\underline{x}$ as follows:

$$
C(\underline{x}, M)^{\bullet}: 0 \longrightarrow C^{0} \stackrel{d^{0}}{\longrightarrow} C^{1} \longrightarrow \cdots \longrightarrow C^{c} \stackrel{d^{c}}{\longrightarrow} C^{c+1} \longrightarrow \cdots \stackrel{d^{n-1}}{\longrightarrow} C^{n} \stackrel{d^{n}}{\longrightarrow} 0 .
$$

Since $c=\operatorname{cd}(\mathfrak{a}, M)=\operatorname{grade}(\mathfrak{a}, M)$, in light of [2, Theorems 5.1 .19 and 6.2.7], $H^{j}\left(C(\underline{x}, M)^{\bullet}\right)=0$ for all $j \neq c$. Hence, we have the exact sequences

$$
0 \longrightarrow C^{0} \stackrel{d^{0}}{\longrightarrow} C^{1} \longrightarrow \cdots \longrightarrow C^{c-2} \stackrel{d^{c-2}}{\longrightarrow} C^{c-1} \longrightarrow L^{c-1} \longrightarrow 0
$$

and

$$
0 \longrightarrow K^{c} \longrightarrow C^{c} \stackrel{d^{c}}{\longrightarrow} C^{c+1} \longrightarrow \cdots \longrightarrow C^{n-1} \stackrel{d^{n-1}}{\longrightarrow} C^{n} \longrightarrow 0 .
$$

So, we can deduce the exact sequences

$$
0 \longrightarrow K^{c} \longrightarrow C^{c} \longrightarrow L^{c} \longrightarrow 0
$$

and

$$
0 \longrightarrow L^{j} \longrightarrow C^{j+1} \longrightarrow L^{j+1} \longrightarrow 0,
$$

for all $0 \leqslant j \leqslant n-1$ with $j \neq c-1$. Note that $L^{n}=0$ and, if $c \neq 0$, we have $L^{0}=C^{0}=$ $M$. Now, by applying the long exact sequence $\mathcal{R}^{j} F(-)$ on the exact sequence (2.9), in conjunction with Lemma 2.1, we have the isomorphisms

$$
\begin{aligned}
\mathcal{R}^{i} F\left(L^{c}\right) & \cong \mathcal{R}^{i-1} F\left(L^{c+1}\right) \\
& \cong \mathcal{R}^{i-2} F\left(L^{c+2}\right) \\
& \cong \cdots \\
& \cong \mathcal{R}^{i+c-n+1} F\left(L^{n-1}\right) \\
& \cong \mathcal{R}^{i+c-n} F\left(L^{n}\right)=0
\end{aligned}
$$

for all $i \in \mathbb{N}_{0}$. So, applying the functor $\mathcal{R}^{j} F(-)$ on the exact sequence (2.8), together with Lemma 2.1, ensures that

$$
\mathcal{R}^{i} F\left(K^{c}\right) \cong \mathcal{R}^{i} F(M) \quad \text { when } c=0
$$

and

$$
\mathcal{R}^{i} F\left(K^{c}\right)=0 \quad \text { when } c \neq 0,
$$


for all $i \in \mathbb{N}_{0}$. Now, since by [2, Theorem 5.1.19], $H_{\mathfrak{a}}^{c}(M) \cong K^{c} / L^{c-1}$, we have the exact sequence

$$
0 \longrightarrow L^{c-1} \longrightarrow K^{c} \longrightarrow H_{\mathfrak{a}}^{c}(M) \longrightarrow 0
$$

and so, when $c=0$, in view of the isomorphism (2.10), for all $i \in \mathbb{N}_{0}$, one can deduce the isomorphism

$$
\mathcal{R}^{i} F\left(H_{\mathfrak{a}}^{c}(M)\right) \cong \mathcal{R}^{i} F(M),
$$

as desired. Also, (2.11) and the exact sequence (2.12) induce the isomorphism

$$
\mathcal{R}^{i} F\left(H_{\mathfrak{a}}^{c}(M)\right) \cong \mathcal{R}^{i+1} F\left(L^{c-1}\right)
$$

when $c \neq 0$. Now, in view of Lemma 2.1, by applying the long exact sequence $\mathcal{R}^{j} F(-)$ on the exact sequence (2.9), for all $i \in \mathbb{N}_{0}$, we have the isomorphisms

$$
\begin{aligned}
\mathcal{R}^{i+1} F\left(L^{c-1}\right) & \cong \mathcal{R}^{i+2} F\left(L^{c-2}\right) \\
& \cong \mathcal{R}^{i+3} F\left(L^{c-3}\right) \\
& \cong \cdots \\
& \cong \mathcal{R}^{i+c-1} F\left(L^{1}\right) \\
& \cong \mathcal{R}^{i+c} F\left(L^{0}\right) \\
& =\mathcal{R}^{i+c} F(M) .
\end{aligned}
$$

So the result follows from (2.13).

SECond Proof. Since $c=\operatorname{cd}(\mathfrak{a}, M)=\operatorname{grade}(\mathfrak{a}, M)$, we have $H_{\mathfrak{a}}^{c}(M) \cong \mathcal{R} \Gamma_{\mathfrak{a}}(M)[c]$. Hence,

$$
\mathcal{R}^{i} F\left(H_{\mathfrak{a}}^{c}(M)\right) \cong \mathcal{R}^{i} F\left(\mathcal{R} \Gamma_{\mathfrak{a}}(M)[c]\right) \cong \mathcal{R}^{i+c}\left(F \cdot \Gamma_{\mathfrak{a}}\right)(M) \cong \mathcal{R}^{i+c} F(M)
$$

This concludes the proof.

\section{Applications for local cohomology modules}

In this section, we provide some applications of Theorem 2.5. The next result gives interesting isomorphisms.

Theorem 3.1. Let $M$ be a finitely generated $R$-module, a be an ideal of $R$ such that $\mathfrak{a} M \neq M$ and $c:=\operatorname{cd}(\mathfrak{a}, M)=\operatorname{grade}(\mathfrak{a}, M)$. Then, for every ideal $\mathfrak{b}$ of $R$ with $\mathfrak{b} \supseteq \mathfrak{a}:$

(i) $H_{\mathfrak{b}}^{i}\left(H_{\mathfrak{a}}^{c}(M)\right) \cong H_{\mathfrak{b}}^{i+c}(M)$ and

(ii) $\operatorname{Ext}_{R}^{i}\left(R / \mathfrak{b}, H_{\mathfrak{a}}^{c}(M)\right) \cong \operatorname{Ext}_{R}^{i+c}(R / \mathfrak{b}, M)$

for all $i \in \mathbb{N}_{0}$.

Proof. The results follow immediately from Theorem 2.5 when we replace the functor $F$ by the $\mathfrak{b}$-torsion functors $\Gamma_{\mathfrak{b}}(-)$ and $\operatorname{Hom}_{R}(R / \mathfrak{b},-)$. 
Recall that an ideal $\mathfrak{a}$ of $R$ is a cohomological complete intersection if height ${ }_{R} \mathfrak{a}=$ $\operatorname{cd}(\mathfrak{a}, R)$. In [4], M. Hellus and P. Schenzel, by applying the spectral sequence methods on a certain truncation complex (cf. [4, Definition 2.1]) proved the following corollary.

Corollary 3.2 (See [4, Corollary 2.3 and Lemma 2.6]). Let $(R, \mathrm{~m})$ be a Gorenstein local ring and $\mathfrak{a}$ be a cohomological complete intersection ideal of $R$. Set $c:=\operatorname{cd}(\mathfrak{a}, R)$ and $d:=\operatorname{dim}_{R} R / \mathfrak{a}$. Then:

(i) $H_{\mathrm{m}}^{d}\left(H_{\mathrm{a}}^{c}(R)\right) \cong E(R / \mathrm{m})$;

(ii) $\operatorname{Ext}_{R}^{d}\left(R / \mathrm{m}, H_{\mathfrak{a}}^{c}(R)\right) \cong E(R / \mathrm{m})$; and

(iii) $H_{\mathfrak{m}}^{i}\left(H_{\mathfrak{a}}^{c}(R)\right)=0=\operatorname{Ext}_{R}^{i}\left(R / \mathfrak{m}, H_{\mathfrak{a}}^{c}(R)\right)$ for all $i \neq d$.

Proof. Since $R$ is Gorenstein, $\operatorname{dim}_{R} R=$ height $_{R} \mathfrak{a}+\operatorname{dim}_{R} R / \mathfrak{a}=c+d$. Hence $H_{\mathrm{m}}^{c+d}(R) \cong$ $E(R / \mathrm{m})$ and $H_{\mathrm{m}}^{i}(R)=0$ for all $i \neq c+d$. Also, in view of the main theorem in [1], $\operatorname{Ext}_{R}^{c+d}(R / \mathrm{m}, R) \cong E(R / \mathrm{m})$ and $\operatorname{Ext}_{R}^{i}(R / \mathrm{m}, R)=0$ for all $i \neq c+d$. The results now follow from Theorem 3.1.

The following corollary was proved in the implications (i) $\Rightarrow$ (ii) and (i) $\Rightarrow$ (v) in [4, Theorem 3.1].

Corollary 3.3. Let a be a cohomological complete intersection ideal of a Gorenstein local ring $R$. Set $c:=\operatorname{cd}(\mathfrak{a}, R)$. Then, for all $\mathfrak{p}$ containing a:

$$
H_{\mathfrak{p} R_{\mathfrak{p}}}^{h(\mathfrak{p})}\left(H_{\mathfrak{a} R_{\mathfrak{p}}}^{c}\left(R_{\mathfrak{p}}\right)\right) \cong E\left(R_{\mathfrak{p}} / \mathfrak{p} R_{\mathfrak{p}}\right)
$$

(ii) $\operatorname{Ext}_{R_{\mathfrak{p}}}^{h(\mathfrak{p})}\left(R_{\mathfrak{p}} / \mathfrak{p} R_{\mathfrak{p}}, H_{\mathfrak{a} R_{\mathfrak{p}}}^{c}\left(R_{\mathfrak{p}}\right)\right) \cong E\left(R_{\mathfrak{p}} / \mathfrak{p} R_{\mathfrak{p}}\right)$, and

(iii) $H_{\mathfrak{p} R_{\mathfrak{p}}}^{i}\left(H_{\mathfrak{a} R_{\mathfrak{p}}}^{c}\left(R_{\mathfrak{p}}\right)\right)=0=\operatorname{Ext}_{R_{\mathfrak{p}}}^{i}\left(R_{\mathfrak{p}} / \mathfrak{p} R_{\mathfrak{p}}, H_{\mathfrak{a} R_{\mathfrak{p}}}^{c}\left(R_{\mathfrak{p}}\right)\right)$ for all $i \neq h(\mathfrak{p})$,

where $h(\mathfrak{p})=\operatorname{dim}_{R_{\mathfrak{p}}} R_{\mathfrak{p}}-$ height $_{R} \mathfrak{a}$.

\section{Endomorphism rings}

In this section we establish a homomorphism from $\operatorname{End}\left(H_{\mathfrak{a}}^{c}(M)\right)$ to $\operatorname{End}\left(H_{\mathfrak{b}}^{c}(M)\right)$, where $\mathfrak{a}$ and $\mathfrak{b}$ are ideals of $R$ such that $\mathfrak{b} \supseteq \mathfrak{a}$ and $c:=\operatorname{grade}(\mathfrak{a}, M)$. To this end, we first present an application of Theorem 2.3 which is needed later. Note that this result was proved in [8, Lemma 2.2(d)] whenever $M:=R$ is Gorenstein local, and in [6, Theorem 2.6] whenever $M:=R$ is an arbitrary commutative Noetherian ring.

Theorem 4.1. Let $c \in \mathbb{N}_{0}$ and $\mathfrak{a}$ be an ideal of $R$ such that $\mathfrak{a} M \neq M$ and $c \leqslant \operatorname{grade}(\mathfrak{a}, M)$. Then for all a-torsion $R$-modules $T$, we have the isomorphism

$$
\operatorname{Hom}_{R}\left(T, H_{\mathfrak{a}}^{c}(M)\right) \cong \operatorname{Ext}_{R}^{c}(T, M) .
$$

In particular,

$$
\operatorname{End}\left(H_{\mathfrak{a}}^{c}(M)\right) \cong \operatorname{Ext}_{R}^{c}\left(H_{\mathfrak{a}}^{c}(M), M\right) .
$$

Proof. The result immediately follows from Theorem 2.3 when we replace the functor $F$ by the a-torsion functors $\operatorname{Hom}_{R}(T,-)$ and $\operatorname{Hom}_{R}\left(H_{\mathfrak{a}}^{c}(M),-\right)$. 
Let $\mathfrak{a}$ and $\mathfrak{b}$ be ideals of a Gorenstein local ring $R$ such that $\mathfrak{b} \supseteq \mathfrak{a}$ and $c:=$ height $_{R} \mathfrak{a}=$ height $_{R} \mathfrak{b}$. Schenzel [8, Theorem 1.2(a)] showed that there exists a natural homomorphism $\operatorname{End}\left(H_{\mathfrak{a}}^{c}(R)\right) \longrightarrow \operatorname{End}\left(H_{\mathfrak{b}}^{c}(R)\right)$. Here, by using Theorem 4.1, we extend the above result as follows.

THEOREM 4.2. Let $\mathfrak{a}$ and $\mathfrak{b}$ be ideals of an arbitrary commutative Noetherian ring $R$ such that $\mathfrak{b} \supseteq \mathfrak{a}, \mathfrak{a} M \neq M$ and $c:=\operatorname{grade}(\mathfrak{a}, M)$. Then:

(i) we have a monomorphism from $H_{\mathfrak{b}}^{c}(M)$ to $H_{\mathfrak{a}}^{c}(M)$; and

(ii) there exists a natural homomorphism from $\operatorname{End}\left(H_{\mathfrak{a}}^{c}(M)\right)$ to $\operatorname{End}\left(H_{\mathfrak{b}}^{c}(M)\right)$.

Proof. (i) In light of Corollary 2.4(i), if we set $F:=\Gamma_{\mathfrak{b}}(-)$, we have the isomorphism

$$
\Gamma_{\mathfrak{b}}\left(H_{\mathfrak{a}}^{c}(M)\right) \cong H_{\mathfrak{b}}^{c}(M) .
$$

So, we have a monomorphism

$$
0 \longrightarrow H_{\mathfrak{b}}^{c}(M) \longrightarrow H_{\mathfrak{a}}^{c}(M)
$$

as desired.

(ii) Applying the long exact sequence $\operatorname{Ext}_{R}^{c}(-, M)$ to (4.1) deduces the natural homomorphism

$$
\operatorname{Ext}_{R}^{c}\left(H_{\mathfrak{a}}^{c}(M), M\right) \longrightarrow \operatorname{Ext}_{R}^{c}\left(H_{\mathfrak{b}}^{c}(M), M\right)
$$

Now Theorem 4.1 completes the proof.

Remark 4.3. By using the proofs of [8, Theorem 1.2(a)] and Theorem 4.2, it is easy to see that if $R$ is a Gorenstein local ring, the natural homomorphisms described in [8, Theorem 1.2(a)] and Theorem 4.2 coincide.

Lemma 4.4. Let $U$ be an $R$-linear contravariant functor from $C(R)$ to itself. Suppose that $\mathfrak{a}$ is an ideal of $R$ and $M$ is a finitely generated $R$-module such that:

(i) $\mathcal{R}^{i} U\left(M_{z}\right)=0$ for all $z \in \mathfrak{a}$;

(ii) $\mathfrak{a} M \neq M$; and

(iii) $c:=\operatorname{cd}(\mathfrak{a}, M)=\operatorname{grade}(\mathfrak{a}, M)$.

Then

$$
\mathcal{R}^{c} U\left(H_{\mathfrak{a}}^{c}(M)\right) \cong \mathcal{R}^{0} U(M)
$$

Proof. First, suppose that $c \geqslant 1$ and that $\left\{x_{1}, \ldots, x_{n}\right\}$ is a generating set of $\mathfrak{a}$. Consider the Čech complex $C(\underline{\mathrm{x}}, M)^{\bullet}$ of $M$ with respect to $\underline{\mathrm{x}}:=x_{1}, \ldots, x_{n}$. By using a method similar to the one we used in the first proof of Theorem 2.5, we have the exact sequences (2.8) and (2.9). Also, by our assumption, $\mathcal{R}^{j} U\left(C^{i}\right)=0$ for all $i, j \in \mathbb{N}$. 
Hence, for $i \in \mathbb{N}$, we have the isomorphisms

$$
\begin{aligned}
\mathcal{R}^{i} U\left(K^{c}\right) & \cong \mathcal{R}^{i+1} U\left(L^{c}\right) \\
& \cong \mathcal{R}^{i+2} U\left(L^{c+1}\right) \\
& \cong \cdots \\
& \cong \mathcal{R}^{i+n-c} U\left(L^{n-1}\right) \\
& \cong \mathcal{R}^{i+n-c+1} U\left(L^{n}\right)=0 .
\end{aligned}
$$

Thus

$$
\mathcal{R}^{i} U\left(K^{c}\right)=0 \text { for all } i \in \mathbb{N} .
$$

Also, by [2, Theorem 5.1.19], $H_{\mathfrak{a}}^{c}(M) \cong K^{c} / L^{c-1}$, and so we have the exact sequence

$$
0 \longrightarrow L^{c-1} \longrightarrow K^{c} \longrightarrow H_{\mathfrak{a}}^{c}(M) \longrightarrow 0 .
$$

Whenever $c=1,(4.2)$ and (4.3) imply the exact sequence

$$
\mathcal{R}^{0} U\left(K^{1}\right) \longrightarrow \mathcal{R}^{0} U\left(L^{0}\right) \longrightarrow \mathcal{R}^{1} U\left(H_{\mathfrak{a}}^{1}(M)\right) \longrightarrow 0 .
$$

Also, by applying the functor $\mathcal{R}^{j} U(-)$ to the exact sequence (2.8) and (2.9), we have the isomorphisms

$$
\mathcal{R}^{0} U\left(K^{1}\right) \cong \mathcal{R}^{1} U\left(L^{1}\right) \cong \mathcal{R}^{2} U\left(L^{2}\right) \cong \cdots \cong \mathcal{R}^{n} U\left(L^{n}\right)=0 .
$$

Thus $\mathcal{R}^{1} U\left(H_{\mathfrak{a}}^{1}(M)\right) \cong \mathcal{R}^{0} U\left(L^{0}\right) \cong \mathcal{R}^{0} U(M)$. So we may assume that $c \geqslant 2$. Now the exact sequences (4.3) and (2.9) in conjunction with (4.2) induce the isomorphisms

$$
\begin{aligned}
\mathcal{R}^{c} U\left(H_{\mathfrak{a}}^{c}(M)\right) & \cong \mathcal{R}^{c-1} U\left(L^{c-1}\right) \\
& \cong \mathcal{R}^{c-2} U\left(L^{c-2}\right) \\
& \cong \cdots \\
& \cong \mathcal{R}^{1} U\left(L^{1}\right) .
\end{aligned}
$$

Moreover, (2.9) implies the isomorphism $\mathcal{R}^{1} U\left(L^{1}\right) \cong \mathcal{R}^{0} U\left(L^{0}\right)$. Thus $\mathcal{R}^{c} U\left(H_{\mathfrak{a}}^{c}(M)\right) \cong$ $\mathcal{R}^{0} U\left(L^{0}\right) \cong \mathcal{R}^{0} U(M)$.

In the case where $c=0$, by slight modifications in the first part of the above arguments, we can show that there exist the exact sequences (2.9) and

$$
0 \longrightarrow H_{\mathfrak{a}}^{0}(M) \longrightarrow M \longrightarrow L^{0} \longrightarrow 0
$$

Hence we have the exact sequence

$$
0 \longrightarrow \mathcal{R}^{0} U\left(L^{0}\right) \longrightarrow \mathcal{R}^{0} U(M) \longrightarrow \mathcal{R}^{0} U\left(H_{\mathfrak{a}}^{0}(M)\right) \longrightarrow \mathcal{R}^{1} U\left(L^{0}\right)
$$

and the isomorphisms

$$
\mathcal{R}^{1} U\left(L^{0}\right) \cong \mathcal{R}^{2} U\left(L^{1}\right) \cong \cdots \cong \mathcal{R}^{n} U\left(L^{n-1}\right) \cong \mathcal{R}^{n} U\left(L^{n}\right)=0 .
$$


Thus

$$
\mathcal{R}^{1} U\left(L^{0}\right)=0 .
$$

Also (2.9) implies the exact sequence

$$
\mathcal{R}^{0} U\left(C^{1}\right) \longrightarrow \mathcal{R}^{0} U\left(L^{0}\right) \longrightarrow \mathcal{R}^{1} U\left(L^{1}\right) .
$$

As we have shown in the first part of the above arguments, $\mathcal{R}^{1} U\left(L^{1}\right)=0$. Moreover, it follows from our assumption that $\mathcal{R}^{0} U\left(C^{1}\right)=0$. Hence $\mathcal{R}^{0} U\left(L^{0}\right)=0$. The result now follows from (4.4) and (4.5).

Theorem 4.5. Let $c \in \mathbb{N}_{0}$ and $M$ be a finitely generated $R$-module. Suppose that $\mathfrak{a}$ is an ideal of $R$ such that $\mathfrak{a} M \neq M$ and $c:=\operatorname{cd}(\mathfrak{a}, M)=\operatorname{grade}(\mathfrak{a}, M)$. Then

$$
\operatorname{End}\left(H_{\mathfrak{a}}^{c}(M)\right) \cong \operatorname{Hom}_{R}(M, M) \text {. }
$$

Proof. Consider the functors $F(-):=\operatorname{Hom}_{R}\left(H_{\mathfrak{a}}^{c}(M),-\right)$ and $U(-):=\operatorname{Hom}_{R}(-, M)$. By Theorem 2.3 and Lemma 4.4, we have the isomorphisms

$$
\begin{aligned}
\operatorname{End}\left(H_{\mathfrak{a}}^{c}(M)\right) & \cong \mathcal{R}^{c} F(M) \\
& \cong \mathcal{R}^{c} U\left(H_{\mathfrak{a}}^{c}(M)\right) \\
& \cong \mathcal{R}^{0} U(M) \\
& \cong \operatorname{Hom}_{R}(M, M) .
\end{aligned}
$$

This concludes the proof.

Suppose that $(R, \mathrm{~m})$ is a Noetherian local complete ring and $\mathfrak{a}$ a proper ideal of $R$ such that $c:=\operatorname{grade}(\mathfrak{a}, R)=\operatorname{cd}(\mathfrak{a}, R)$. Let $E$ be the injective hull of $R / \mathrm{m}$. Since $R$ is complete, for every $z \in \mathfrak{a}$ we have the isomorphisms

$$
\operatorname{Ext}_{R}^{i}\left(R_{z}, R\right) \cong \operatorname{Ext}_{R}^{i}\left(R_{z}, \operatorname{Hom}_{R}(E, E)\right) \cong \operatorname{Hom}_{R}\left(\operatorname{Tor}_{i}^{R}\left(E, R_{z}\right), E\right)=0 .
$$

Then, by Theorem $4.5, \operatorname{End}\left(H_{\mathfrak{a}}^{c}(R)\right) \cong R$. This means that Theorem 4.5 is an improved form of [5, Theorem 2.2].

\section{Acknowledgements}

The authors would like to thank the referee for interest in the subject, careful reading of and constructive comments on an earlier version of this paper, which resulted in an improved presentation.

\section{References}

[1] H. Bass, 'On the ubiquity of Gorenstein rings', Math. Z. 82 (1983), 18-29.

[2] M. Brodmann and R. Y. Sharp, Local Cohomology: An Algebraic Introduction with Geometric Applications, Cambridge Studies in Advanced Mathematics, 60 (Cambridge University Press, Cambridge, 1998). 
[3] M. Hellus, 'Local cohomology and complete intersections of rank 1', Preprint, arXiv:math.Ac/0509652 v1.

[4] M. Hellus and P. Schenzel, 'On cohomological complete intersection', J. Algebra 320(10) (2008), 3733-3748.

[5] M. Hellus and J. Stückrad, 'On endomorphism rings of local cohomology', Proc. Amer. Math. Soc. 130 (2008), 2333-2341.

[6] K. Khashyarmanesh, 'On the endomorphism rings of local cohomology modules', Canad. Math. Bull. 53(4) (2010), 667-673.

[7] K. Khashyarmanesh and M. Yassi, 'On the finiteness property of generalized local cohomology modules', Algebra Colloq. 12(2) (2005), 293-300.

[8] P. Schenzel, 'On endomorphism rings and dimensions of local cohomology modules', Proc. Amer. Math. Soc. 137(4) (2009), 1315-1322.

K. KHASHYARMANESH, Department of Pure Mathematics, Ferdowsi University of Mashhad, PO Box 1159-91775, Mashhad, Iran

e-mail: khashyar@ipm.ir

F. KHOSH-AHANG, Department of Mathematics, Ilam University, PO Box 69315-516, Ilam, Iran

e-mail: Khoshahang@ferdowsi.um.ac.ir 